\title{
Synthesis and structure of metal complexes containing zwitterionic $N$-hydroxyimidazole ligands
}

\author{
Suaad Abuskhuna ${ }^{\text {a }}$, Malachy McCann ${ }^{\mathrm{a}, *}$, John Briody ${ }^{\mathrm{a}}$, Michael Devereux ${ }^{\mathrm{b}}$, \\ Kevin Kavanagh ${ }^{c}$, Nijhuma Kayal ${ }^{\mathrm{d}}$, Vickie McKee ${ }^{\mathrm{d}}$ \\ ${ }^{a}$ Chemistry Department, National University of Ireland Maynooth, Maynooth, Co. Kildare, Ireland \\ ${ }^{\mathrm{b}}$ Dublin Institute of Technology, Cathal Brugha Street, Dublin, Ireland \\ ${ }^{\mathrm{c}}$ Biology Department, National University of Ireland Maynooth, Maynooth, Co. Kildare, Ireland \\ ${ }^{d}$ Chemistry Department, Loughborough University, Loughborough, Leics. LE11 3TU, UK \\ Received 7 May 2007; received in revised form 8 June 2007; accepted 9 June 2007 \\ Available online 13 August 2007
}

\begin{abstract}
$\mathrm{Cu}(\mathrm{II})$ and $\mathrm{Zn}$ (II) complexes of $N$-hydroxyimidazoles were synthesised by reacting simple metal perchlorate salts with the imidazole ligand in alcohol and formulated with a metal:ligand ratio of 1:2. The X-ray crystal structures of five complexes (four $\mathrm{Cu}(\mathrm{II})$ and one $\mathrm{Zn}(\mathrm{II}))$ were obtained and each showed the two trans, $N$-hydroxyimidazole ligands forming six-membered, chelate rings with the metal. Both of the NO chelating, neutral $N$-hydroxyimidazole ligands are in the zwitterion form, with the uncoordinated imidazole imine $\mathrm{N}$ atom being protonated and the oxime $\mathrm{O}$ atom deprotonated. In the solid state the complexes form hydrogen-bonded supramolecular structures.
\end{abstract}

(c) 2007 Elsevier Ltd. All rights reserved.

Keywords: $N$-hydroxyimidazole; Metal complex; Zwitterion; X-ray structure; Supramolecular

\section{Introduction}

The imidazole diazole ring is commonly found in highly significant endogenous biomolecules, including biotin, the essential amino acid histidine, histamine, the pilocarpine alkaloids [1] and other alkaloids, which have been shown to exhibit interesting biological activities such as antimicrobial, anticryptococcal, inhibition of nitric oxide synthase, and cytotoxic activities [2]. Several imidazole derivatives, such as cimetidine, etomidate and ketoconazole, are commonly prescribed pharmaceutical drugs [3,4]. $N$-hydroxyimidazoles have been reported [5] to possess occidiostatic, anthelmintic, herbicidal, insecticidal, nematocidal, fungicidal and bacteriostatic activities. Antihypertensive activity was observed in some $N$-hydroxyimidazole-5-methanamine derivatives [6], and $N$-hydroxyimidazole-3-oxides have

\footnotetext{
* Corresponding author. Tel.: +353 1 7083767; fax: +35317083815.

E-mail address: mmcann@nuim.ie (M. McCann).
}

been investigated as reactivators of organophosphateinhibited acetylcholinesterase enzymes [7]. Recently [8,9], we have described the synthesis and structures of a number of metal complexes containing bidentate, bis-imidazole ligands. A common structural feature of these ligands is that the two imidazole rings are linked by a single spacer tetrahedral carbon. In the present work, five new $N$ hydroxyimidazole ligands (Fig. 1) were prepared and these have a direct link between the two heteroxyclic rings (imidazole-imidazole or imidazole-pyridine). The $N$-hydroxyimidazole ligands were complexed to $\mathrm{Cu}(\mathrm{II})$ and $\mathrm{Zn}(\mathrm{II})$ centres. Five complexes were crystallographically characterised, revealing that the chelating ligands exist in a novel zwitterion form.

\section{Results and discussion}

$\mathrm{Cu}(\mathrm{II})$ and $\mathrm{Zn}$ (II) complexes of the $N$-hydroxyimidazoles were synthesised by reacting the simple metal perchlo- 

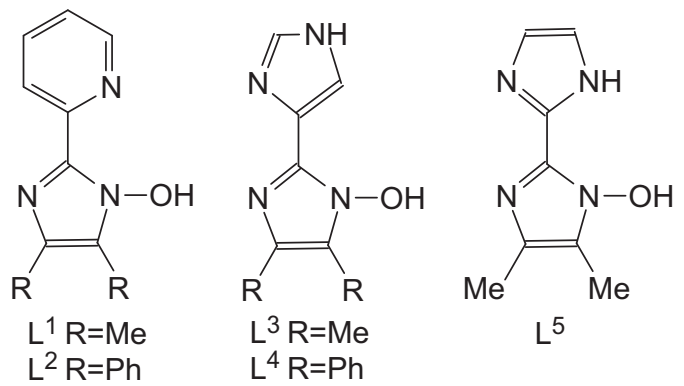

Fig. 1. $N$-hydroxyimidazole ligands.

rate salt with the imidazole ligand in alcohol. The complexes were soluble in a variety of organic solvents and insoluble in water. The complexes formulated with a metal:ligand ratio of $1: 2$ and were characterised by spectroscopy \{IR and NMR (where appropriate)\}, microanalysis and X-ray crystallography.

The X-ray crystal structure of $\left[\mathrm{Cu}\left(\mathrm{L}^{1}\right)_{2}\right]\left(\mathrm{ClO}_{4}\right)_{2} \cdot \mathrm{H}_{2} \mathrm{O}$ (Figs. 2a-2c, Tables 1 and 2) shows the $\mathrm{Cu}(\mathrm{II})$ ion sitting at a centre of symmetry and with tetragonal geometry. The two $\mathrm{L}^{1}$ ligands form six-membered, chelate rings with the metal and are trans to each other. Both of the NO chelating, neutral $N$-hydroxyimidazole ligands have tautomerised and are in the zwitterion form (Fig. 3), with the uncoordinated imidazole imine $\mathrm{N}$ atom being protonated and the oxime $\mathrm{O}$ atom deprotonated. There are also long axial bonds to the two perchlorate anions and the solvate water molecule makes three hydrogen bonds to the protonated amine, the oxime $\mathrm{O}$ atom and to a perchlorate $\mathrm{O}$ atom. The two heterocyclic rings within each ligand are titled at $13.8(1)^{\circ}$ to one another. There is $\pi-\pi$ stacking (Fig. 2b) creating an infinite two-dimensional network sheet structure. The sheets are linked through the hydrogen bonds between the water molecules and the ligands resulting in the formation of channels which host the water molecules (Fig. 2c).

The three other $\mathrm{Cu}$ (II) complexes and one $\mathrm{Zn}$ (II) complex all contain two NO chelating zwitterionic $N$-hydroxy-

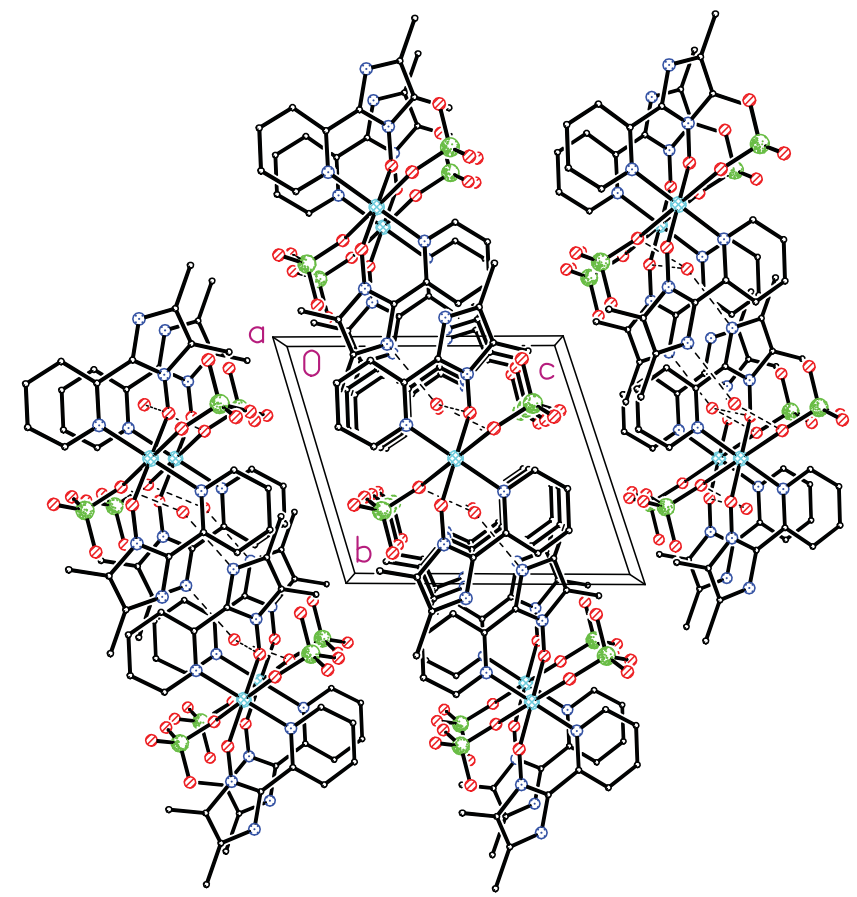

Fig. 2b. $\pi-\pi$ stacking in $\left[\mathrm{Cu}\left(\mathrm{L}^{1}\right)_{2}\right]\left(\mathrm{ClO}_{4}\right)_{2} \cdot \mathrm{H}_{2} \mathrm{O}$.

imidazole ligands (Figs. 4-7, Tables 1 and 2) and have core structures similar to $\left[\mathrm{Cu}\left(\mathrm{L}^{1}\right)_{2}\right]\left(\mathrm{ClO}_{4}\right)_{2} \cdot \mathrm{H}_{2} \mathrm{O}$. The three anhydrous $\mathrm{Cu}(\mathrm{II})$ complexes have approximate square planar coordination geometry. In the case of $\left[\mathrm{Cu}\left(\mathrm{L}^{2}\right)_{2}\right]\left(\mathrm{ClO}_{4}\right)_{2}$ there are additional hydrogen bonding interactions between the protonated imine $\mathrm{N}$ atoms and the perchlorate oxygen atoms stitching neighbouring complexes into infinite chains (for example see Fig. 4). Intermolecular bridging perchlorates and $\pi-\pi$ stacking orders the $\left[\mathrm{Cu}\left(\mathrm{L}^{3}\right)_{2}\right]\left(\mathrm{ClO}_{4}\right)_{2}$ units complexes into a supramolecular array (Fig. 5). In the case of $\left[\mathrm{Cu}\left(\mathrm{L}^{5}\right)_{2}\right]\left(\mathrm{ClO}_{4}\right)_{2}$, weak ' $\mathrm{Cu}-$ perchlorate-protonated imine' interactions create chains of complex dications (Fig. 7b).

In $\left[\mathrm{Zn}\left(\mathrm{L}^{4}\right)_{2} \mathrm{MeOH}\right]\left(\mathrm{ClO}_{4}\right)_{2} \cdot \mathrm{MeOH}$ the cation is a centrosymmetric dimer (Fig. 6a) where the two halves (Fig. 6b) are linked by two bridging deprotonated hydroxyl

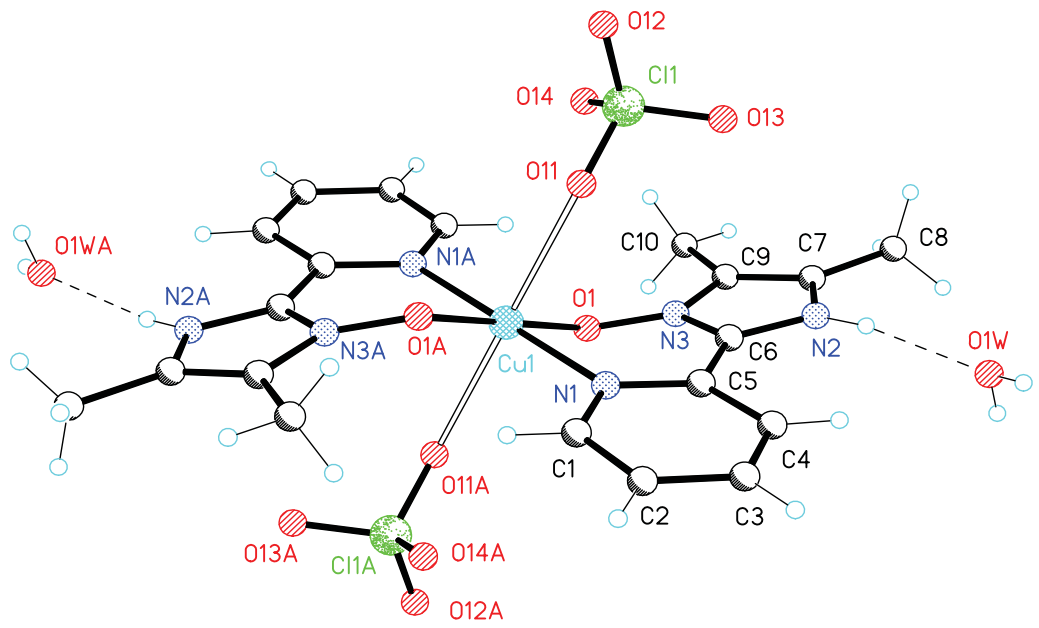

Fig. 2a. X-ray structure of $\left[\mathrm{Cu}\left(\mathrm{L}^{1}\right)_{2}\right]\left(\mathrm{ClO}_{4}\right)_{2} \cdot \mathrm{H}_{2} \mathrm{O}$. 


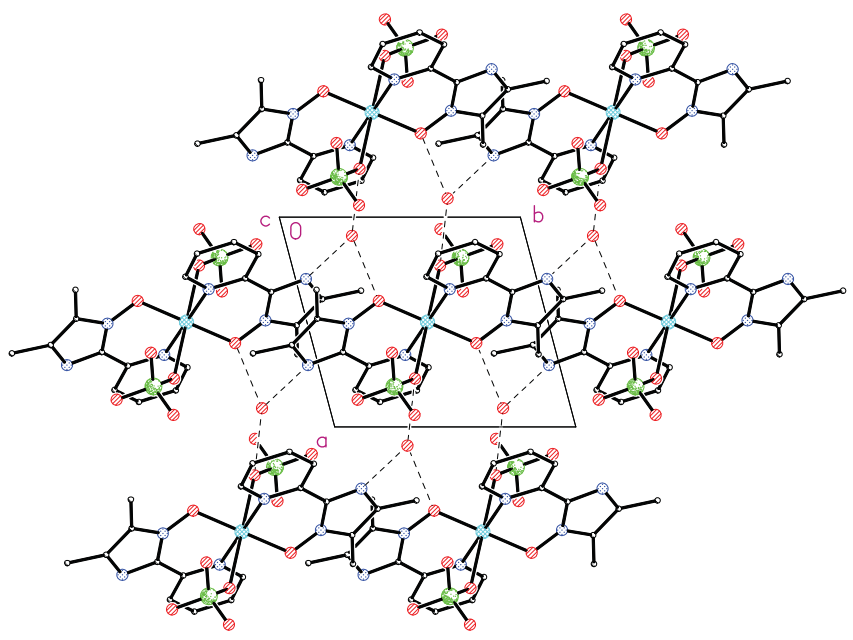

Fig. 2c. Hydrogen-bonded water molecules in the channels formed in $\left[\mathrm{Cu}\left(\mathrm{L}^{1}\right)_{2}\right]\left(\mathrm{ClO}_{4}\right)_{2} \cdot \mathrm{H}_{2} \mathrm{O}$.

$\mathrm{O}^{-}$atoms $(\mathrm{O} 2)$. The $\mathrm{Zn}(\mathrm{II})$ ions are six-coordinate, the sixth ligand being a methanol solvate molecule. There is extensive hydrogen bonding network involving the coordinated methanol, the methanol of solvation, the perchlorate anion and the hydrogen of the protonated amine $\mathrm{N}$ atom. This three-dimensional coordination polymer structure is shown in Fig. 6c.

To our knowledge, the above five complexes are the first documented examples of $N$-hydroxyimidazoles tautomerising to form zwitterions upon complexation to a metal ion and, as such, represent a novel class of ligand.

\section{Experimental}

Chemicals were purchased from commercial sources and, unless specified, were used without further purification. $N$-Hydroxyimidazoles were prepared by reacting monoximes with aldehydes and ammonia [10,11]. Infrared spectra of solids (in a $\mathrm{KBr}$ matrix) were recorded in the region $4000-400 \mathrm{~cm}^{-1}$ on a Nicolet FT-IR Impact 400D infrared spectrometer. ${ }^{1} \mathrm{H}$ NMR spectra were run as solutions in DMSO- $d_{6}$ on a Bruker Avance $300 \mathrm{MHz}$ instrument. Microanalytical data were provided by the Microanalytical Laboratory, National University of Ireland, Cork, Ireland. X-ray crystallographic data (Table 3) were collected at $150 \mathrm{~K}$ on a Bruker SMART 1000 diffractometer using Mo K $\alpha$ radiation $(\lambda=0.71073 \AA)$. The structures were solved by direct methods and refined by
Table 2

Hydrogen bonds $\left(\AA\right.$ and $\left.^{\circ}\right)$

\begin{tabular}{lcccc}
\hline $\mathrm{D}-\mathrm{H} \ldots \mathrm{A}$ & $d(\mathrm{D}-\mathrm{H})$ & $d(\mathrm{H} \ldots \mathrm{A})$ & $d(\mathrm{D} \ldots \mathrm{A})$ & $\langle(\mathrm{DHA})$ \\
\hline$\left[\mathrm{Cu}\left(\mathrm{L}^{1}\right)_{2}\right]\left(\mathrm{ClO}_{4}\right)_{2} \cdot \mathrm{H}_{2} \mathrm{O}$ & & & & \\
$\mathrm{N}(2)-\mathrm{H}(2 \mathrm{~N}) \ldots \mathrm{O}(1 \mathrm{~W})$ & $0.84(3)$ & $1.91(3)$ & $2.723(3)$ & $162(3)$ \\
$\mathrm{O}(1 \mathrm{~W})-\mathrm{H}(1 \mathrm{WA}) \ldots \mathrm{O}(11) \# 1$ & $0.75(3)$ & $2.16(3)$ & $2.911(2)$ & $177(3)$ \\
$\mathrm{O}(1 \mathrm{~W})-\mathrm{H}(2 \mathrm{WB}) \ldots \mathrm{O}(1) \# 2$ & $0.78(3)$ & $2.08(3)$ & $2.831(2)$ & $161(3)$ \\
{$\left[\mathrm{Cu}\left(\mathrm{L}^{2}\right)_{2}\right]\left(\mathrm{ClO}_{4}\right)_{2} \cdot \mathrm{H}_{2} \mathrm{O}$} & & & & \\
$\mathrm{N}(2)-\mathrm{H}(2 \mathrm{~N}) \ldots \mathrm{O}(11)$ & $0.82(3)$ & $2.01(3)$ & $2.8284(19)$ & $177(2)$ \\
{$\left[\mathrm{Cu}\left(\mathrm{L}^{3}\right)_{2}\right](\mathrm{ClO})_{2}$} & & & & \\
$\mathrm{~N}(2)-\mathrm{H}(2 \mathrm{~N}) \ldots \mathrm{O}(14) \# 3$ & $0.79(3)$ & $2.11(3)$ & $2.895(2)$ & $169(3)$ \\
$\mathrm{N}(3)-\mathrm{H}(3 \mathrm{~N}) \ldots \mathrm{O}(11)$ & $0.84(3)$ & $2.06(3)$ & $2.859(2)$ & $157(3)$ \\
{$\left[\mathrm{Zn}\left(\mathrm{L}^{4}\right)_{2} \mathrm{MeOH}\right](\mathrm{ClO})_{4} \cdot \mathrm{MeOH}$} & & & & \\
$\mathrm{N}(12)-\mathrm{H}(12) \ldots \mathrm{O}(23) \# 4$ & 0.88 & 2.02 & $2.831(3)$ & 153.6 \\
$\mathrm{~N}(13)-\mathrm{H}(13) \ldots \mathrm{O}(12)$ & 0.88 & 2.03 & $2.898(3)$ & 169.9 \\
$\mathrm{~N}(22)-\mathrm{H}(22) \ldots \mathrm{O}(11) \# 5$ & 0.88 & 2.02 & $2.894(3)$ & 172.2 \\
$\mathrm{~N}(23)-\mathrm{H}(23) \ldots \mathrm{O}(22) \# 6$ & 0.88 & 2.21 & $3.012(3)$ & 152.1 \\
$\mathrm{~N}(23)-\mathrm{H}(23) \ldots \mathrm{O}(14) \# 7$ & 0.88 & 2.57 & $3.182(3)$ & 126.9 \\
$\mathrm{O}(40)-\mathrm{H}(40) \ldots \mathrm{O}(50)$ & 0.85 & 1.87 & $2.717(3)$ & 172.6 \\
$\mathrm{O}(50)-\mathrm{H}(50) \ldots \mathrm{O}(24)$ & 0.85 & 2.23 & $3.032(3)$ & 158.4 \\
$\left.\left[\mathrm{Cu}\left(\mathrm{L}^{5}\right)_{2}\right](\mathrm{ClO})_{4}\right)_{2}$ & & & & \\
$\mathrm{~N}(2)-\mathrm{H}(2 \mathrm{~N}) \ldots \mathrm{O}(11)$ & 0.86 & 1.95 & $2.787(3)$ & 163.1 \\
$\mathrm{~N}(3)-\mathrm{H}(3 \mathrm{~N}) \ldots \mathrm{O}(12)$ & 0.88 & 2.12 & $2.946(4)$ & 154.7 \\
\hline
\end{tabular}

Symmetry transformations used to generate equivalent atoms: $\# 1-x$, $-y+2,-z+1 ; \# 2-x+1,-y+2,-z+1 ; \# 3-x-1,-y+2,-z+1$; $\# 4-x,-y,-z+1 ; \# 5 x, y+1, z ; \# 6-x,-y+1,-z+1 ; \# 7-x+1$, $-y+1,-z+1$.

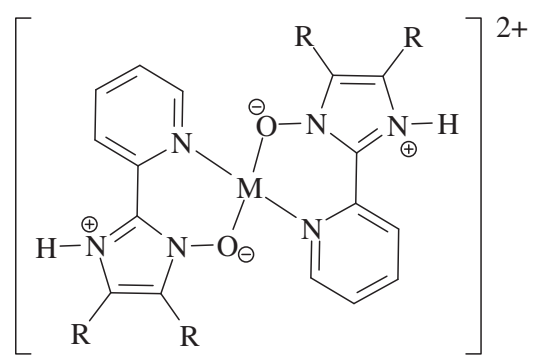

Fig. 3. Zwitterionic form of $N$-hydroxyimidazole ligands chelated to the metal $\left(\mathrm{M}^{2+}\right)$ centre.

full-matrix least-squares on $F^{2}$ using all the reflections. All the non-hydrogen atoms were refined anisotropically and hydrogen atoms bonded to carbon were inserted at calculated positions using a riding model. Hydrogen atoms bonded to $\mathrm{O}$ or $\mathrm{N}$ were located from difference maps and refined with fixed, isotropic thermal parameters,

Table 1

Selected bond lengths $(\AA)$ and angles $\left(^{\circ}\right)$

\begin{tabular}{lllll}
\hline Complex & {$\left[\mathrm{Cu}\left(\mathrm{L}^{1}\right)_{2}\right]\left(\mathrm{ClO}_{4}\right)_{2} \cdot \mathrm{H}_{2} \mathrm{O}$} & {$\left[\mathrm{Cu}\left(\mathrm{L}^{2}\right)_{2}\right]\left(\mathrm{ClO}_{4}\right)_{2} \cdot \mathrm{H}_{2} \mathrm{O}$} & {$\left[\mathrm{Cu}\left(\mathrm{L}^{3}\right)_{2}\right]\left(\mathrm{ClO}_{4}\right)_{2}$} & {$\left[\mathrm{Zn}\left(\mathrm{L}^{4}\right)_{2} \mathrm{MeOH}\right](\mathrm{ClO})_{2} \cdot \mathrm{MeOH}$} \\
\hline $\mathrm{M}-\mathrm{O}$ & $1.9217(15)$ & $1.9305(12)$ & $1.9297(13)$ & $2.0409(15), 2.0941(15)$ \\
M-N & $2.0523(17)$ & $2.0140(14)$ & $1.9479(15)$ & $2.0550(19), 2.0783(19)$ \\
O-M-N & $90.61(7)$ & $90.25(5)$ & $93.14(6)$ & $90.26(7), 87.77(7)$ \\
O-M-N\#1 & $89.39(7)$ & $89.75(5)$ & $86.86(6)$ & $89.87(7), 92.67(7)$ \\
Symmetry ops \#1 & $-x+1,-y+1,-z+1$ & $-x+2,-y+1,-z$ & $-x+1,-y+1,-z+1$ & none \\
\hline
\end{tabular}




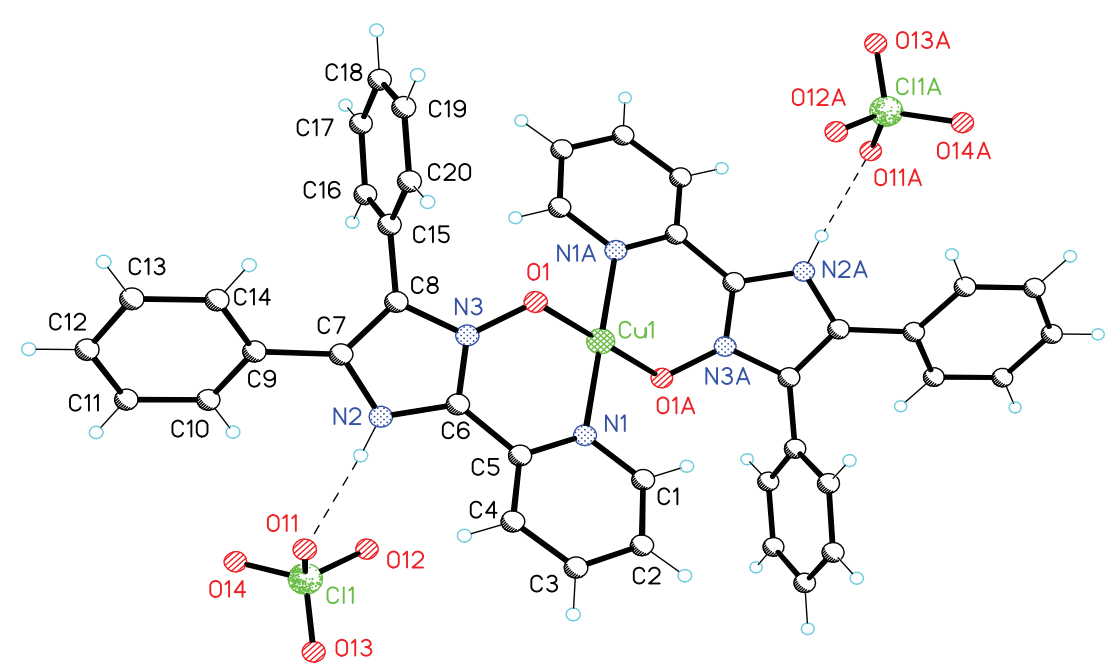

Fig. 4a. X-ray structure of $\left[\mathrm{Cu}\left(\mathrm{L}^{2}\right)_{2}\right]\left(\mathrm{ClO}_{4}\right)_{2}$.

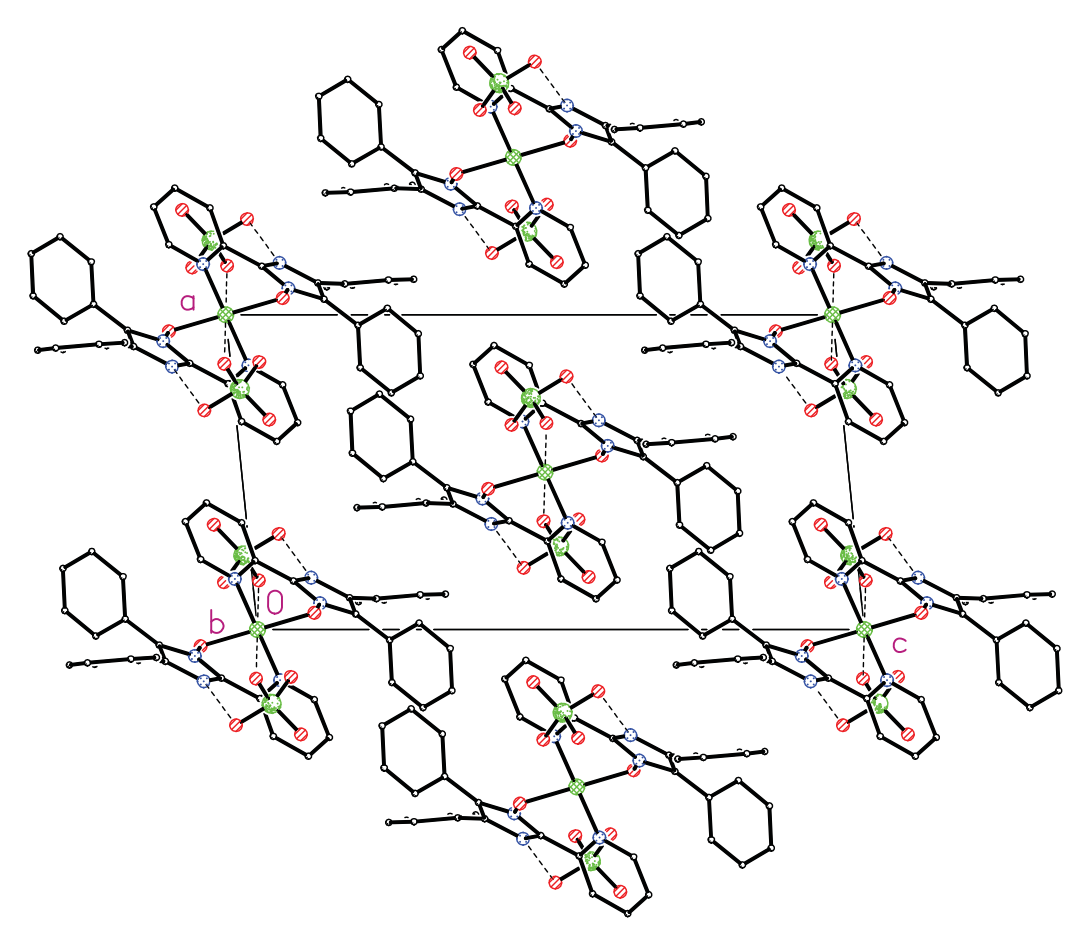

Fig. 4b. Hydrogen bonding interactions forming infinite chains of $\left[\mathrm{Cu}\left(\mathrm{L}^{2}\right)_{2}\right]\left(\mathrm{ClO}_{4}\right)_{2}$ complexes.

except for $\left[\mathrm{Zn}\left(\mathrm{L}^{4}\right)_{2} \mathrm{MeOH}\right]\left(\mathrm{ClO}_{4}\right)_{2} \cdot \mathrm{MeOH}$ (where all the $\mathrm{H}$ atoms were inserted at calculated positions) and $\left[\mathrm{Cu}\left(\mathrm{L}^{5}\right)_{2}\right]\left(\mathrm{ClO}_{4}\right)_{2}$ (where they were located and not further refined).

\section{1. $\left[\mathrm{Cu}\left(\mathrm{L}^{\mathrm{I}}\right)_{2}\right]\left(\mathrm{ClO}_{4}\right)_{2} \cdot \mathrm{H}_{2} \mathrm{O}$}

A solution of $\mathrm{Cu}\left(\mathrm{ClO}_{4}\right)_{2} \cdot 6 \mathrm{H}_{2} \mathrm{O}$ and $\mathrm{L}^{1}(1: 2 \mathrm{~mol}$ ratio) in acetonitrile was refluxed for $4 \mathrm{~h}$ and then left to stand overnight. The precipitated green complex was recrystallised from a mixture of acetonitrile and ethanol. Yield $=81 \%$. The complex was soluble in hot acetonitrile and insoluble in water. Anal. Calc. for $\mathrm{C}_{20} \mathrm{H}_{24} \mathrm{~N}_{6} \mathrm{O}_{11} \mathrm{Cl}_{2} \mathrm{Cu}$ : C, 36.46; $\mathrm{H}$,
3.67; N, 12.76. Found: C, 36.50; H, 3.29; N, 12.32\%. IR: $3456,3121,1644,1605,1573,1541,1491,1434,1316$, $1288,1231,1117,969,928,823,782,625,484 \mathrm{~cm}^{-1}$.

\section{2. $\left[\mathrm{Cu}\left(\mathrm{L}^{2}\right)_{2}\right]\left(\mathrm{ClO}_{4}\right)_{2} \cdot \mathrm{H}_{2} \mathrm{O}$}

This green complex was prepared and recrystallised in a similar way to $\left[\mathrm{Cu}\left(\mathrm{L}^{1}\right)_{2}\right]\left(\mathrm{ClO}_{4}\right)_{2} \cdot \mathrm{H}_{2} \mathrm{O}$. Yield $=56 \%$. The complex was soluble in hot acetonitrile and insoluble in water. Anal. Calc. for $\mathrm{C}_{40} \mathrm{H}_{32} \mathrm{~N}_{6} \mathrm{O}_{11} \mathrm{Cl}_{2} \mathrm{Cu}$ : C, 52.97; $\mathrm{H}$, 3.56; N, 9.27. Found: C, 52.92; H, 3.31; N, 9.33\%. IR: $3456,3126,1605,1576,1544,1480,1427,1309,1210$, $1096,925,825,764,702,623,545 \mathrm{~cm}^{-1}$. 
a
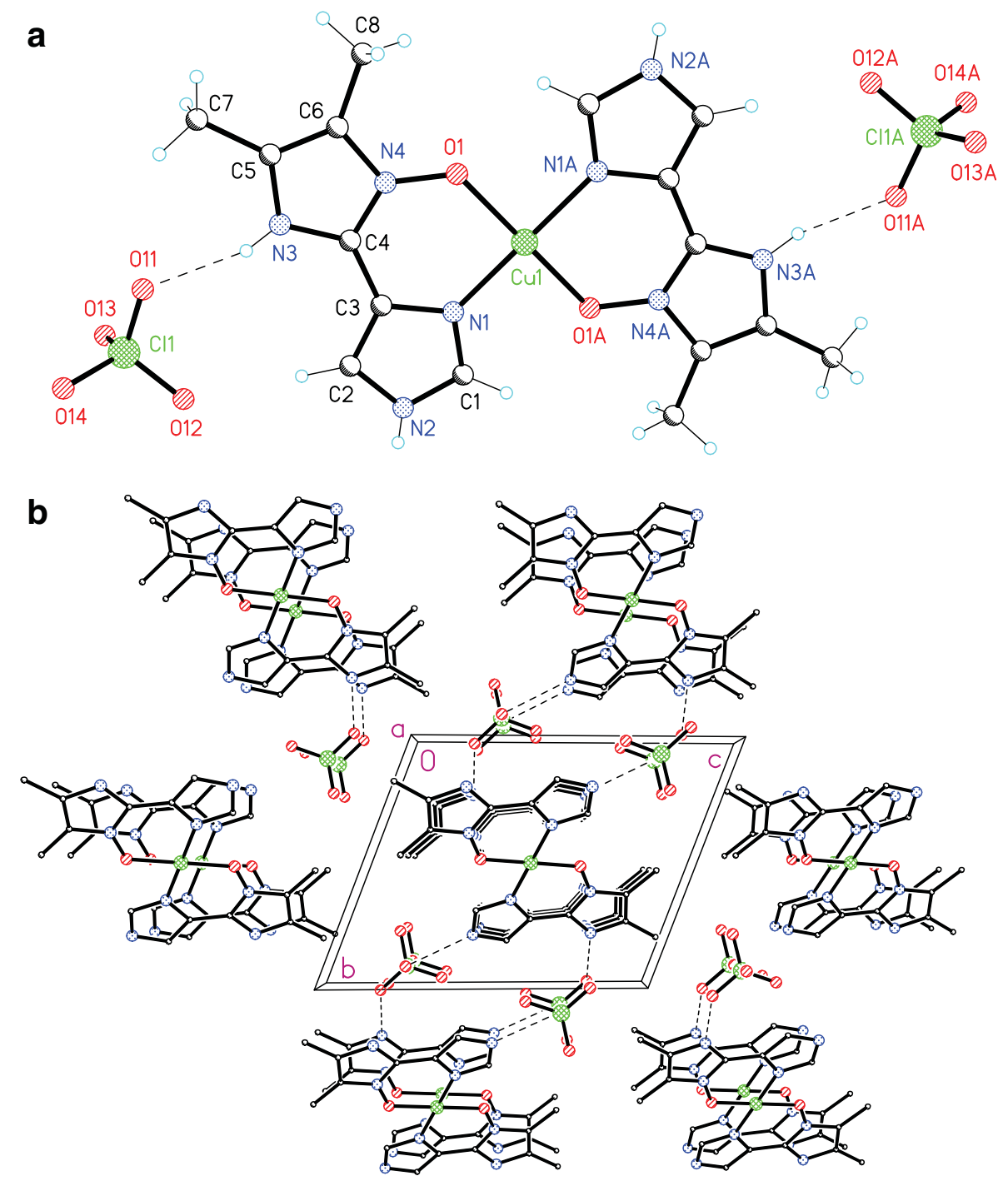

Fig. 5. X-ray structure of $\left[\mathrm{Cu}\left(\mathrm{L}^{3}\right)_{2}\right]\left(\mathrm{ClO}_{4}\right)_{2}$.

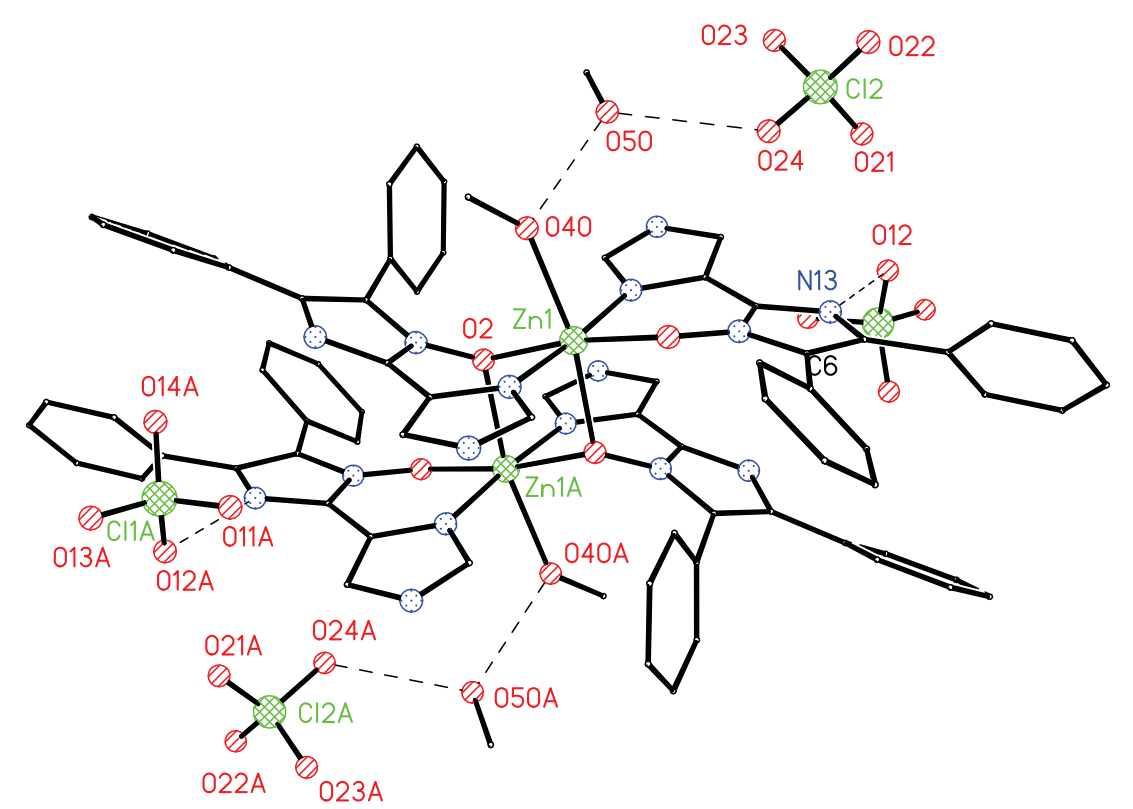

Fig. 6a. X-ray structure of dimeric $\left[\mathrm{Zn}\left(\mathrm{L}^{4}\right)_{2} \mathrm{MeOH}\right]\left(\mathrm{ClO}_{4}\right)_{2} \cdot \mathrm{MeOH}$. 


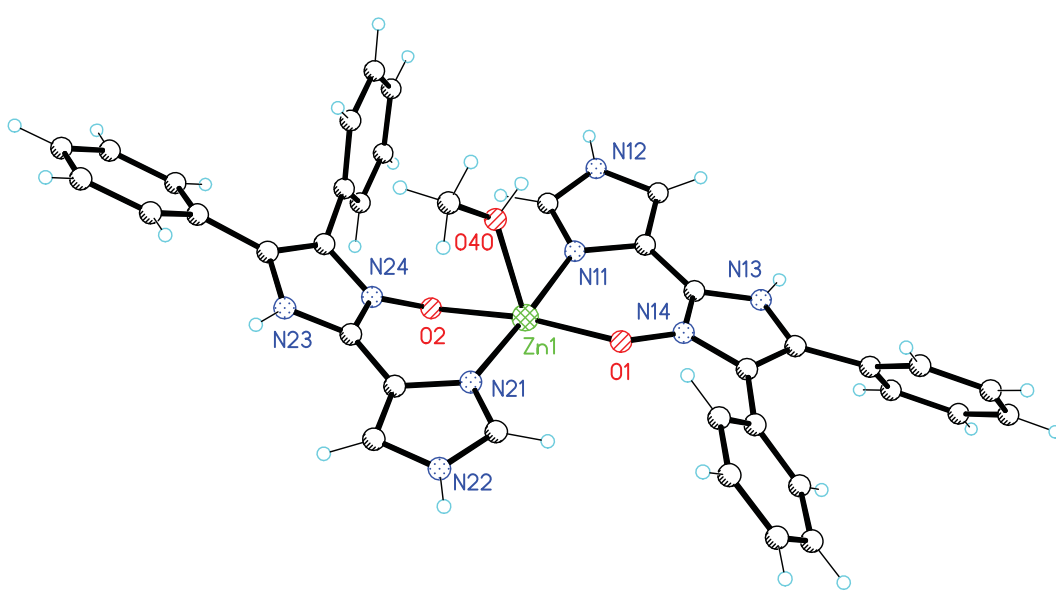

Fig. 6b. One half of dimeric $\left[\mathrm{Zn}\left(\mathrm{L}^{4}\right)_{2} \mathrm{MeOH}\right]\left(\mathrm{ClO}_{4}\right)_{2} \cdot \mathrm{MeOH}$.

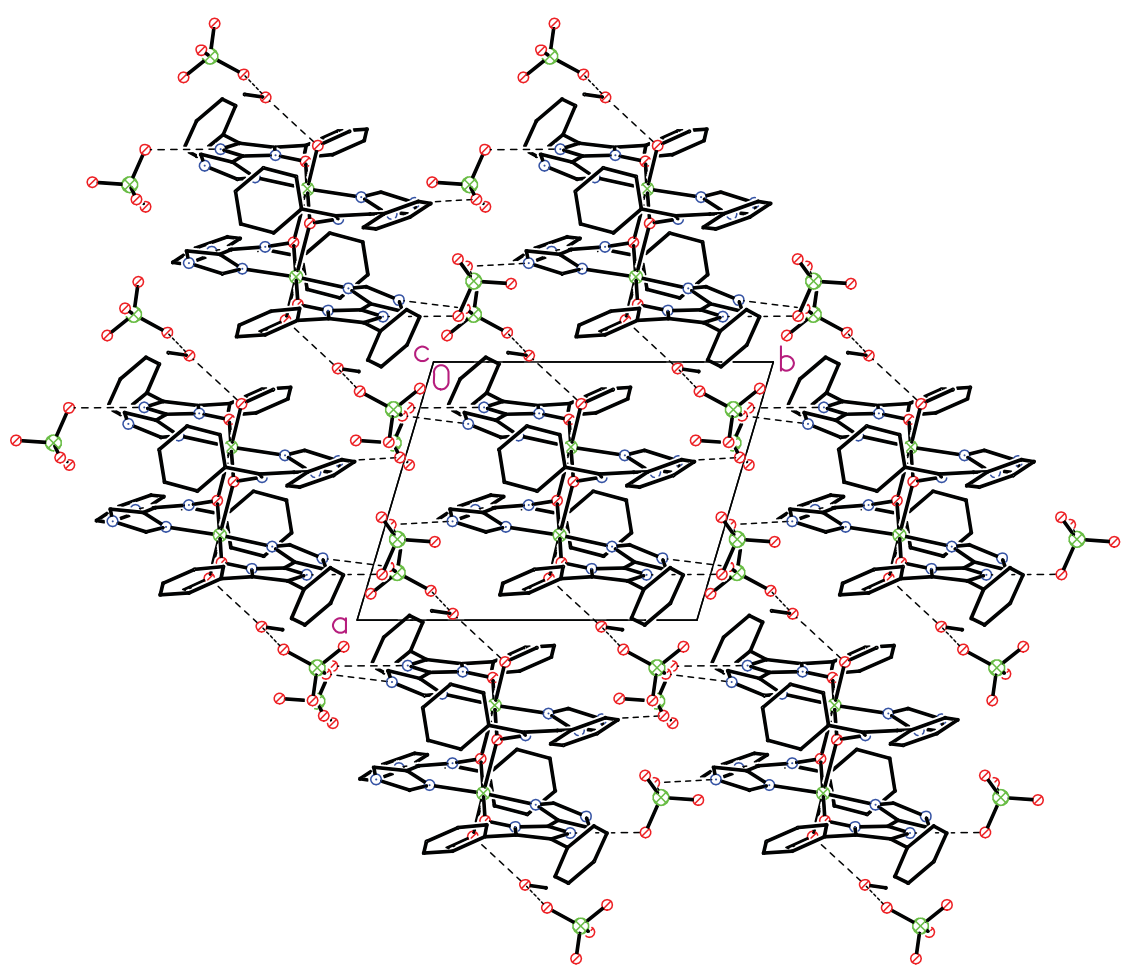

Fig. 6c. Extensive hydrogen bonding network in $\left[\mathrm{Zn}\left(\mathrm{L}^{4}\right)_{2} \mathrm{MeOH}\right]\left(\mathrm{ClO}_{4}\right)_{2} \cdot \mathrm{MeOH}$.

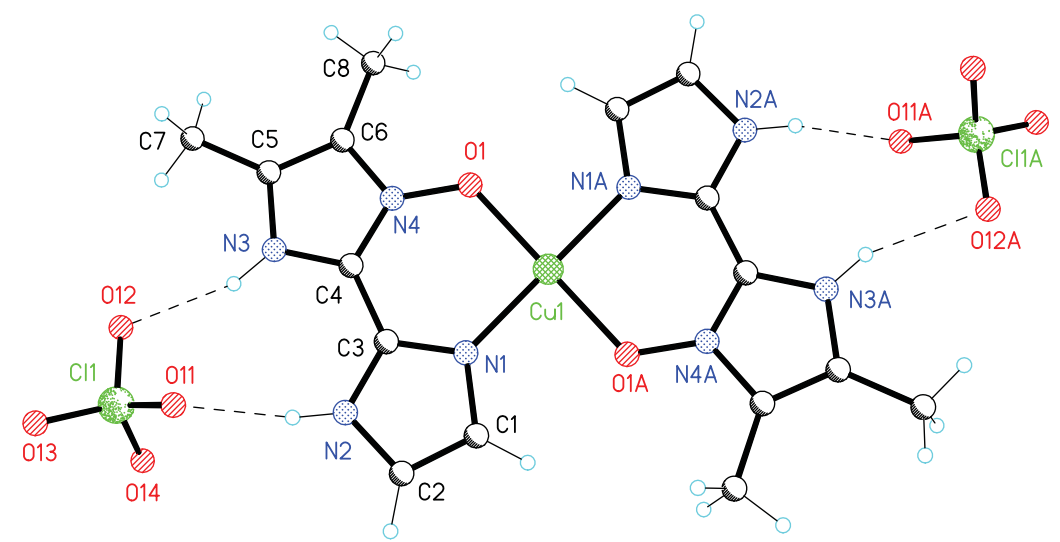

Fig. 7a. X-ray structure of $\left[\mathrm{Cu}\left(\mathrm{L}^{5}\right)_{2}\right]\left(\mathrm{ClO}_{4}\right)_{2}$. 


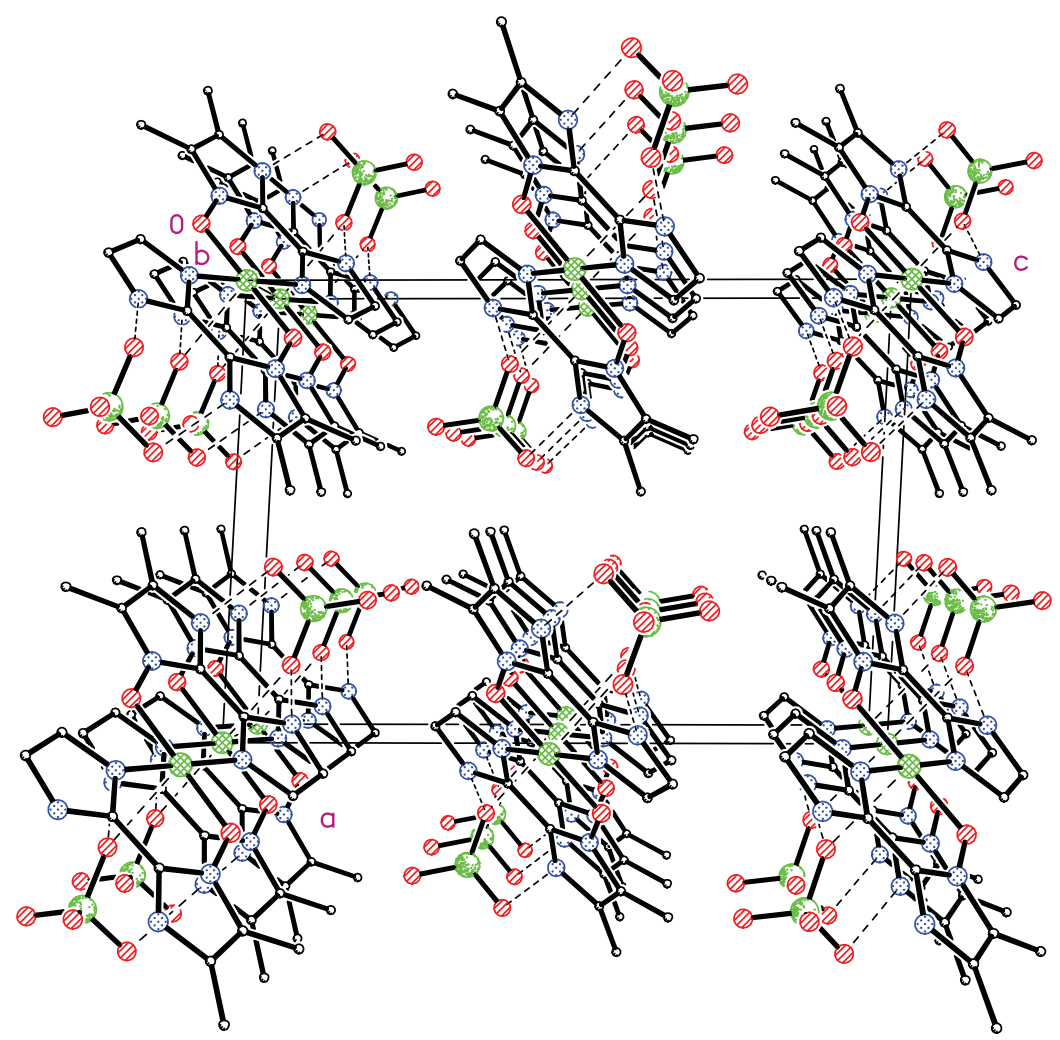

Fig. 7b. Chains of $\left[\mathrm{Cu}\left(\mathrm{L}^{5}\right)_{2}\right]\left(\mathrm{ClO}_{4}\right)_{2}$ formed through ' $\mathrm{Cu}$-perchlorate-protonated imine' interactions.

Table 3

Crystal data and structure refinements

\begin{tabular}{|c|c|c|c|c|c|}
\hline Complex & {$\left[\mathrm{Cu}\left(\mathrm{L}^{1}\right)_{2}\right]\left(\mathrm{ClO}_{4}\right)_{2} \cdot \mathrm{H}_{2} \mathrm{O}$} & {$\left[\mathrm{Cu}\left(\mathrm{L}^{2}\right)_{2}\right]\left(\mathrm{ClO}_{4}\right)_{2}$} & {$\left[\mathrm{Cu}\left(\mathrm{L}^{3}\right)_{2}\right]\left(\mathrm{ClO}_{4}\right)_{2}$} & {$\left[\mathrm{Zn}\left(\mathrm{L}^{4}\right)_{2} \mathrm{MeOH}\right]\left(\mathrm{ClO}_{4}\right)_{2} \cdot \mathrm{MeOH}$} & {$\left[\mathrm{Cu}\left(\mathrm{L}^{5}\right)_{2}\right]\left(\mathrm{ClO}_{4}\right)_{2}$} \\
\hline Empirical formula & $\mathrm{C}_{20} \mathrm{H}_{26} \mathrm{Cl}_{2} \mathrm{CuN}_{6} \mathrm{O}_{12}$ & $\mathrm{C}_{40} \mathrm{H}_{30} \mathrm{Cl}_{2} \mathrm{CuN}_{6} \mathrm{O}_{10}$ & $\mathrm{C}_{16} \mathrm{H}_{20} \mathrm{Cl}_{2} \mathrm{CuN}_{8} \mathrm{O}_{10}$ & $\mathrm{C}_{38} \mathrm{H}_{36} \mathrm{Cl}_{2} \mathrm{~N}_{8} \mathrm{O}_{12} \mathrm{Zn}$ & $\mathrm{C}_{16} \mathrm{H}_{20} \mathrm{Cl}_{2} \mathrm{CuN}_{8} \mathrm{O}_{10}$ \\
\hline Formula weight & 676.91 & 889.14 & 618.84 & 933.02 & 618.84 \\
\hline Crystal system & triclinic & monoclinic & triclinic & triclinic & monoclinic \\
\hline Space group & $P \overline{1}$ & $P 2_{1} / n$ & $P \overline{1}$ & $P \overline{1}$ & $P 2_{1} / c$ \\
\hline$a(\AA)$ & $7.7461(10)$ & $10.0378(8)$ & $4.8193(5)$ & $10.1946(7)$ & $10.8501(9)$ \\
\hline$b(\AA)$ & $9.0192(12)$ & $9.8351(8)$ & $9.9883(10)$ & $12.9732(9)$ & $6.9525(6)$ \\
\hline$c(\AA)$ & $9.9153(13)$ & $19.2452(15)$ & $12.3209(12)$ & $15.7194(11)$ & $15.5483(13)$ \\
\hline$\alpha\left({ }^{\circ}\right)$ & $71.879(2)$ & 90 & $110.492(2)$ & $97.800(1)$ & 90 \\
\hline$\beta\left({ }^{\circ}\right)$ & $84.956(2)$ & $95.7500(10)$ & $95.627(2)$ & $92.308(1)$ & $93.044(1)$ \\
\hline$\gamma\left({ }^{\circ}\right)$ & $74.375(2)$ & 90 & $93.404(2)$ & $105.961(1)$ & 90 \\
\hline Volume $\left(\AA^{3}\right)$ & $634.03(14)$ & $1890.4(3)$ & $550.08(10)$ & $1973.8(2)$ & $1171.24(17)$ \\
\hline$Z$ & 1 & 2 & 1 & 2 & 2 \\
\hline$D_{\text {calc }}(\mathrm{Mg} / \mathrm{m})$ & 1.773 & 1.562 & 1.868 & 1.570 & 1.755 \\
\hline Reflections collected & 5122 & 15171 & 4690 & 17260 & 9520 \\
\hline $\begin{array}{l}\text { Independent reflections } \\
{\left[R_{\text {int }}\right]}\end{array}$ & $2799[0.0176]$ & $4386[0.0205]$ & $2471[0.0124]$ & $9032[0.0226]$ & $2745[0.0185]$ \\
\hline Data/restraints/parameter & 2799/0/198 & $4386 / 0 / 271$ & $2471 / 0 / 177$ & $9032 / 0 / 552$ & $2745 / 0 / 171$ \\
\hline Goodness-of-fit on $F^{2}$ & 1.024 & 1.031 & 1.073 & 1.022 & 0.896 \\
\hline$R_{1}, w R_{2}[I>2 \sigma(I)]$ & $0.0310,0.0720$ & $0.0317,0.0787$ & $0.0266,0.0664$ & $0.0390,0.0880$ & $0.0370,0.1037$ \\
\hline$R_{1}, w R_{2}$ (all data) & $0.0427,0.0773$ & $0.0418,0.0834$ & $0.0308,0.0685$ & $0.0573,0.0973$ & $0.0444,0.1094$ \\
\hline
\end{tabular}

\section{3. $\left[\mathrm{Cu}\left(\mathrm{L}^{3}\right)_{2}\right]\left(\mathrm{ClO}_{4}\right)_{2}$}

This green complex was prepared and recrystallised in a similar way to $\left[\mathrm{Cu}\left(\mathrm{L}^{1}\right)_{2}\right]\left(\mathrm{ClO}_{4}\right)_{2} \cdot \mathrm{H}_{2} \mathrm{O}$. Yield $=61 \%$. The complex was soluble in hot acetonitrile and insoluble in water. Anal. Calc. for $\mathrm{C}_{16} \mathrm{H}_{20} \mathrm{~N}_{8} \mathrm{O}_{10} \mathrm{Cl}_{2} \mathrm{Cu}$ : C, 31.05; H, 3.26; N, 18.11. Found: C, 31.09; H, 3.10; N, 18.34\%. IR:
$3227,1619,1512,1439,1292,1234,1097,960,928,789$, $760,669,620,473 \mathrm{~cm}^{-1}$.

\section{4. $\left[\mathrm{Zn}\left(\mathrm{L}^{4}\right)_{2} \mathrm{MeOH}\right]\left(\mathrm{ClO}_{4}\right)_{2} \cdot \mathrm{MeOH}$}

This white complex was prepared in the same manner as $\left[\mathrm{Cu}\left(\mathrm{L}^{1}\right)_{2}\right]\left(\mathrm{ClO}_{4}\right)_{2} \cdot \mathrm{H}_{2} \mathrm{O}$ and was recrystallised from a 
mixture of methanol and acetonitrile. Yield $=83 \%$. The complex was soluble in hot methanol and acetonitrile and insoluble in water. Anal. Calc. for $\mathrm{C}_{36} \mathrm{H}_{28} \mathrm{~N}_{8} \mathrm{O}_{10} \mathrm{Cl}_{2} \mathrm{Zn}$ : C, 49.76; H, 3.25; N, 12.89. Found: C, 49.20; H, 3.49; N, 12.76\%. ${ }^{1} \mathrm{H}$ NMR: 7.00-7.15 (aromatic H, m), 7.57-7.69 (imidazole H, m). IR: 3272, 1646, 1510, 1436, 1301, 1104, $987,926,760,698,655,627,529 \mathrm{~cm}^{-1}$.

\section{5. $\left[\mathrm{Cu}\left(\mathrm{L}^{5}\right)_{2}\right]\left(\mathrm{ClO}_{4}\right)_{2}$}

This green complex was prepared and recrystallised in a similar way to $\left[\mathrm{Cu}\left(\mathrm{L}^{1}\right)_{2}\right]\left(\mathrm{ClO}_{4}\right)_{2} \cdot \mathrm{H}_{2} \mathrm{O}$. Yield $=72 \%$. The complex was soluble in hot acetonitrile and ethanol and insoluble in water. Anal. Calc. for $\mathrm{C}_{16} \mathrm{H}_{20} \mathrm{~N}_{8} \mathrm{O}_{10} \mathrm{Cl}_{2} \mathrm{Cu}$ : C, $31.05 ; \mathrm{H}, 3.26 ; \mathrm{N}, 18.11$. Found: C, 30.74; H, 3.02; N, $17.52 \%$. IR: $3217,1609,1531,1443,1317,1126,1039$, $919,811,767,713,627,473 \mathrm{~cm}^{-1}$.

\section{Acknowledgement}

S. Abuskhuna acknowledges financial support from the Libyan Higher Education Department.

\section{Appendix A. Supplementary material}

CCDC 638219, 638220, 638221, 638222 and 638223 contain the supplementary crystallographic data for $\left[\mathrm{Cu}\left(\mathrm{L}^{1}\right)_{2}\right]\left(\mathrm{ClO}_{4}\right)_{2} \cdot \mathrm{H}_{2} \mathrm{O},\left[\mathrm{Cu}\left(\mathrm{L}^{2}\right)_{2}\right]\left(\mathrm{ClO}_{4}\right)_{2} \cdot \mathrm{H}_{2} \mathrm{O},\left[\mathrm{Cu}\left(\mathrm{L}^{3}\right)_{2}\right]-$
$\left(\mathrm{ClO}_{4}\right)_{2},\left[\mathrm{Zn}\left(\mathrm{L}^{4}\right)_{2} \mathrm{MeOH}\right]\left(\mathrm{ClO}_{4}\right)_{2} \cdot \mathrm{MeOH}$ and $\left[\mathrm{Cu}\left(\mathrm{L}^{5}\right)_{2}\right]-$ $\left(\mathrm{ClO}_{4}\right)_{2}$. These data can be obtained free of charge via http://www.ccdc.cam.ac.uk/conts/retrieving.html, or from the Cambridge Crystallographic Data Centre, 12 Union Road, Cambridge CB2 1EZ, UK; fax: (+44) 1223-336033; or e-mail: deposit@ccdc.cam.ac.uk. Supplementary data associated with this article can be found, in the online version, at doi:10.1016/j.poly.2007.06.034.

\section{References}

[1] M.R. Grimmett, in: A.R. Katritsky, E.F.V. Scriven (Eds.), Comprehensive Heterocyclic Chemistry II, vol. 3, Pergamon, Oxford, 1996, pp. 77-220.

[2] L. De Luca, Curr. Med. Chem. 13 (2006) 1.

[3] J.N. Delgado, W.A. Remers (Eds.), Wilson and Gisvold's Textbook of Organic Medicinal and Pharmaceutical Chemistry, 10th ed., Lippincott-Raven, Philadelphia, PA, 1998

[4] J.R. Coura, S.L. de Castro, Mem. Inst. Oswaldo Cruz 97 (2002) 3.

[5] G.G. Allan, C.S. Chopra, T. Mattila, Pest. Sci. 3 (1972) 153.

[6] H.A. Philipp, L.I. Jirkovsky, Am. Home Product Corp. USA (1980) 6.

[7] P. Franchetti, M. Grifantini, C. Lucarelli, Farmaco-Ed. Sci. 27 (1972) 46.

[8] S. Abuskhuna, M. McCann, J. Briody, M. Devereux, V. McKee, Polyhedron 23 (2004) 1731.

[9] S. Abuskhuna, J. Briody, M. McCann, M. Devereux, K. Kavanagh, J. Barreira Fontecha, V. McKee, Polyhedron 23 (2004) 1249.

[10] S. Abuskhuna, M.Sc. thesis, NUI Maynooth, 2004

[11] K. Akagane, G.G. Allan, Shikizai Kyokaishi 46 (1973) 555. 